\title{
AMPLIFICATION OF WAVES REFLECTED FROM \\ KERR BLACK HOLES
}

\author{
A. A. STAROBINSKY
}

The Landau Institute for Theoretical Physics, Moscow., U.S.S.R.

\begin{abstract}
The effect of amplification of electromagnetic and gravitational waves reflected from a rotating black hole ('superradiance scattering') is investigated. This effect was proposed by Zel'dovich (1971). It leads, as well as the Penrose process, to the energy extraction from a Kerr black hole at the expense of its rotational energy and momentum decrease. The coefficient of wave reflection $R>1$ if $\omega<n \Omega$, where $\omega$ is the wave frequency, $n$-its angular momentum and $\Omega$ is the black hole angular velocity. The value of this effect is not small in the case of gravitational waves, for example, if $l=n=2, \omega \rightarrow n \Omega$ and $a=M$, then $R \approx 2.38$.

There also exists a quantum version of the effect, namely, the one of spontaneous pair creation in the Kerr metric, but this quantum effect is exceedingly small in real astrophysical conditions, because its characteristic time is of the order $G^{2} M^{3} / \hbar c^{4}$, where $M$ is the black hole mass.
\end{abstract}

\section{References}

Zel'dovich, Ya. B.: 1971, Pisma v Zh.E.T.F. (in Russian) 14, 270.

Starobinsky, A. A. and Churilov, S. M.: 1973, Zh.E.T.F. (in Russian) 65, 3. 